\title{
Attenuation compensation in cerebral 3D PET: effect of the attenuation map on absolute and relative quantitation
}

\author{
Habib Zaidi, Marie-Louise Montandon, Daniel O. Slosman \\ Division of Nuclear Medicine, Geneva University Hospital, Geneva 4, Switzerland
}

Received: 21 May 2003 / Accepted: 4 August 2003 / Published online: 22 October 2003

(C) Springer-Verlag 2003

\begin{abstract}
It is generally well accepted that transmission (TX)-based non-uniform attenuation correction can supply more accurate absolute quantification; however, whether it provides additional benefits in routine clinical diagnosis based on qualitative interpretation of 3D brain positron emission tomography (PET) images is still the subject of debate. The aim of this study was to compare the effect of the two major classes of method for determining the attenuation map, i.e. uniform versus non-uniform, using clinical studies based on qualitative assessment as well as absolute and relative quantitative volume of interest-based analysis. We investigated the effect of six different methods for determining the patient-specific attenuation map. The first method, referred to as the uniform fit-ellipse method (UFEM), approximates the outline of the head by an ellipse assuming a constant linear attenuation factor $\left(\mu=0.096 \mathrm{~cm}^{-1}\right)$ for soft tissue. The second, referred to as the automated contour detection method (ACDM), estimates the outline of the head from the emission sinogram. Attenuation of the skull is accounted for by assuming a constant uniform skull thickness $(0.45 \mathrm{~cm})$ within the estimated shape and the correct $\mu$ value $\left(0.151 \mathrm{~cm}^{-1}\right)$ is used. The usual measured transmission method using caesium-137 single-photon sources was used without (MTM) and with segmentation of the TX data (STM). These techniques were finally compared with the segmented magnetic resonance imaging method (SMM) and an implementation of the inferring attenuation distributions method (IADM) based on the digital Zubal head atlas. Several image quality parameters were compared, including absolute and relative quantification indexes, and the correlation between them was checked. The qualitative evaluation showed no significant differences between the different attenuation
\end{abstract}

Habib Zaidi ( $)$

Division of Nuclear Medicine, Geneva University Hospital,

1211 Geneva 4, Switzerland

e-mail: habib.zaidi@hcuge.ch

Tel.: +41-22-3727258, Fax: +41-22-3727169 correction techniques as assessed by expert physicians, with the exception of ACDM, which generated artefacts in the upper edges of the head. The mean squared error between the different attenuation maps was also larger when using this latter method owing to the fact that the current implementation of the method significantly overestimated the head contours on the external slices. Correlation between the mean regional cerebral glucose metabolism (rCGM) values obtained with the various attenuation correction methods and those obtained with the gold standard (MTM) was good, except in the case of ACDM $\left(R^{2}=0.54\right)$. The STM and SMM methods showed the best correlation $\left(R^{2}=0.90\right)$ and the regression lines agreed well with the line of identity. Relative differences in mean rCGM values were in general less than $8 \%$. Nevertheless, ANOVA results showed statistically significant differences between the different methods for some regions of the brain. It is concluded that the attenuation map influences both absolute and relative quantitation in cerebral 3D PET. Transmission-less attenuation correction results in a reduced radiation dose and makes a dramatic difference in acquisition time, allowing increased patient throughput.

Keywords: Positron emission tomography - Brain imaging - Attenuation map - Attenuation correction - Quantification

Eur J Nucl Med Mol Imaging (2004) 31:52-63

DOI 10.1007/s00259-003-1325-8

\section{Introduction}

Attenuation correction is a crucial step in both qualitative assessment and quantitative analysis of reconstructed positron emission tomography (PET) images. Information about patient-specific tissue attenuating properties can be derived from different methods, but is more 
commonly estimated by acquiring an additional preinjection transmission (TX) scan using external sources. Approaches described in the literature based on transmission scanning use either radionuclide positron (germanium-68) or single-photon (caesium-137) emitting sources or alternatively X-ray tubes [1]. Although necessary, this additional procedure complicates the scanner design and increases the radiation dose to staff and patients. Moreover, it substantially increases the overall acquisition time, decreasing patient comfort and scanner throughput and indirectly increasing the costs of PET examinations. Theoretically, measured transmission images introduce the least bias. While the clinical relevance of non-uniform attenuation correction is well established in thoracic imaging, it is still the subject of heated debate in brain scanning, where in most cases clinical diagnosis is based on qualitative assessment of PET images [2, 3, 4, 5, 6]. In principle, brain PET studies simplify this task owing to the homogeneous characteristics of tissue components in the head region [4]. This fact has motivated the development of approximate methods to correct for attenuation with the aim of partially minimising some of the side-effects described above. Calculated methods are at the top of this simplification scheme. Among the techniques that have found widespread use within this class of methods is the interactive contour-drawing method, which consists in manually fitting an ellipse around the scalp from preliminary 2D reconstructions of uncorrected PET images. While easy to implement, manual contour delineation is error prone and operator dependent since inter- and intra-subject variability is unavoidable.

Automatic edge detection algorithms define the external head contour from the emission (EM) sinograms, allowing assignment of known attenuation coefficients to the different tissues considered (e.g. brain and surrounding skull) [7]. More refined algorithms make use of an automated method to compute a three-component attenuation map for brain PET imaging [8]. The algorithm generates an estimated skull image by filtered backprojection of the reciprocal of an emission sinogram whose thickness and radius are estimated from profiles extracted from this image. In general, this type of approach is advantageous in terms of overall acquisition time, and it allows the avoidance of misalignment between EM and TX images. On the other hand, invalid assumption of tissue uniformity might lead to significant activity quantitation bias, especially in regions of high variability, such as air cavities and nasal sinuses. A completely different and relatively new approach overcomes this problem by inferring anatomy from a head atlas [9]. There is, however, a rather important conceptual limitation to this technique, namely the existence of patient-specific anomalies that are obviously not modelled in an atlas obtained from a single or even an average representation of the population. More importantly, successful intramodality image registration strongly relies on many parame- ters, and errors resulting from misregistration could result in significant artefacts. For instance, studies have shown that a co-registration between PET and magnetic resonance (MR) images has an accuracy limit of approximately $2 \mathrm{~mm}$, which is fairly large, considering that structures of only a few millimetres are often of interest. Assuming that a perfect registration could be obtained between MRI and PET, we proposed a new method to construct the attenuation map from co-registered segmented T1-weighted MRI [10].

The challenging issue of assessing differences between the effectiveness of uniform versus non-uniform attenuation maps has been largely studied in single-photon emission tomography (SPET) [2, 11, 12, 13, 14]. Similarly, the paradoxical effect of the complexly shaped skull base surrounding the lower half of the human brain in lowering the mean effective broad-beam attenuation coefficient is well understood in SPET imaging [5, 15, 16]. However, very few studies have been performed to characterise this effect using 3D PET data. Comparisons of more than two methods for determining the attenuation map are rarely found [17], and they generally include combinations with other degrading effects, such as scatter or partial volume effect. The aim of this study was to compare the effect of six different methods belonging the two major classes of method for determining the attenuation map, i.e. non-uniform versus uniform, using clinical 3D brain PET studies based on qualitative assessment as well as absolute and relative quantitative volume of interest (VOI)-based analysis.

\section{Materials and methods}

\section{Methods for determination of the attenuation map}

Uniform fit-ellipse method (UFEM). The first calculated method approximates the outline of the head by a slice-dependent ellipse assuming a constant linear attenuation factor $\left(\mu=0.096 \mathrm{~cm}^{-1}\right)$ for brain tissue [4]. The method is simple but requires an additional preliminary 2D reconstruction of uncorrected PET data, allowing operator interaction to manually draw an ellipse, which in our case was performed by an experienced nuclear medicine technologist.

Automated contour detection method (ACDM). The second calculated method aims at diminishing the subjective effect of operator dependency by using an automatic edge detection algorithm to estimate the external contour of the head from the emission sinograms [7]. Two regions are then identified and assigned corresponding attenuation coefficients: brain tissue and skull. Attenuation of the skull is estimated by assuming a certain uniform skull thickness $(0.45 \mathrm{~cm})$ within the estimated shape and the correct linear attenuation coefficient $\left(\mu=0.151 \mathrm{~cm}^{-1}\right)$ is applied. It is worth emphasising that while the method identifies accurately the head outline in the majority of central slices using a threshold fixed to $15 \%$, it significantly overestimates the contour in the external slices, leading to visible artefacts on the edges of the reconstructed sagittal slices. 
Measured transmission method (MTM). The method used in clinical routine in our department for attenuation correction is based on the acquisition of an additional pre-injection TX scan (10 min) using ${ }^{137} \mathrm{Cs}$ single-photon sources. A thermoplastic face mask is used to limit head motion and for accurate repositioning of patients for the emission scan. The TX data are normalised to a slab phantom scan and corrected for scatter and cross-section variation using a log-linear transformation of the attenuation factors [18]. The images reconstructed with TX-based attenuation correction served as the gold standard for comparative assessment of the different attenuation correction methods.

Segmented transmission method (STM). Segmentation of the transmission data has been traditionally performed when using a short transmission scanning acquisition time with the goal of reducing noise in the associated attenuation-corrected emission data by limiting its propagation through the reconstruction process [19]. In this study, segmentation of the transmission images was performed using an adaptive local histogram-based thresholding technique [20].

Segmented MRI-guided method (SMM). For all patients studied, a high-resolution 3D T1-weighted MRI head scan was also available through the picture archiving and communication system (PACS), which provides an infrastructure for various kinds of medical image retrieval that can be used through the hospital's local area network. The scalp, skull and meninges were removed prior to registration between MRI and PET images using a semi-automated brain extraction tool. This step is recommended when registering intermodality images since the algorithm strongly relies on equivalence between tissue types, and these structures are obviously not present in PET scans. MRI-PET co-registration was performed using the Automatic Image Registration (AIR 3.08) algorithm, consisting in a general linear rescaling [21]. Transformation matrices were recorded and re-applied to the original MR images. A fast implementation of the fuzzy c-means (FCM) segmentation algorithm, already validated for segmentation of transmission images [19], was then used to classify the MRI head tissues into main components in terms of soft tissue contrast. This procedure was followed by appropriate assignment of attenuation coefficients according to the method described by Zaidi et al. [10].

Inferring attenuation distributions method (IADM). This method was originally proposed by Stodilka et al. [9] for SPET brain imaging and has been adapted for PET without any modifications (e.g. adding the bed image to the final attenuation map) [17]. The methodology that we followed was practically the same as that proposed by Stodilka et al.; however, registration of emission images to the three-dimensional Zubal head atlas [22] was best achieved in our case using a non-linear warping algorithm [21], rather than a global rescaling (seven-parameter model) as used in ref. [9]. IADM was derived by registering the brain component of the digital head atlas with a source distribution having specific activities of the grey matter, white matter and ventricles of 4:1:0, respectively, to a preliminary PET reconstruction and then applying the resulting spatial transformation to the full head atlas. The latter was used to construct a patient-specific attenuation map by assigning known attenuation coefficients to different head tissues, similar to the approach followed in SMM [10].

\section{Clinical data acquisition and reconstruction}

As the main focus of this study was comparison of different attenuation correction schemes within subjects, patient selection was limited to those having clinically indicated cerebral PET scans. A total of ten patients admitted for evaluation of surgical relief of their epilepsy were selected from our clinical database and used for comparative assessment of different attenuation correction techniques. All patients included in the study gave their written informed consent for participation. Selection was restricted to patients who had previously undergone a brain MRI scan. The emission study (25 min) started $30 \mathrm{~min}$ after intravenous injection of approximately $222 \mathrm{MBq}$ of fluorine-18 fluorodeoxyglucose (FDG). All data sets were acquired in 3D mode with a maximum acceptance angle corresponding to 17 rings and a span of 7 on an ECAT ART PET scanner (CTI PET Systems, Knoxville, TN) upgraded to use collimated ${ }^{137} \mathrm{Cs}$ single-photon point sources for TX scanning. Acquired projection data were pre-corrected for scatter using the latest numerical implementation of the single-scatter simulation algorithm [23] supplied with the ECAT 7.2 software provided by the scanner manufacturer. Attenuation correction factors were generated by forward projecting the constructed attenuation maps obtained using the six different methods described in the previous section without any extra smoothing. The re-projection algorithm (3DRP) used routinely for reconstruction of clinical brain studies in our division was used (Ramp filter, cut-off frequency 0.35 cycles/pixel), resulting in 47 slices consisting of $128 \times 128$ matrices with a zoom factor of 3 (voxel size, $1.716 \times 1.716 \times 3.375 \mathrm{~mm}^{3}$ ).

Patients' anatomy was defined using a high-resolution 3D T1weighted MR sequence performed on a 1.5-T Eclipse scanner (Philips Medical Systems, Best, The Netherlands). A three-dimensional volumetric acquisition of a T1-weighted gradient echo sequence produced a gapless series of thin sagittal sections (TE/TR, 4.4/15 ms; flip angle, $25^{\circ}$; acquisition matrix, 256 $\times 256 \times 160$; slice thickness, $1.1 \mathrm{~mm}$ ).

\section{Comparative evaluation strategy}

Qualitative and quantitative assessments of differences between images reconstructed using different attenuation correction schemes were carried out by visual assessment performed by trained nuclear medicine physicians and by estimating parameters of clinical interest, including absolute and relative regional cerebral glucose metabolism (rCGM). Error and bias were examined by calculating difference images between MTM and alternative methods for deriving the attenuation map. The mean square error (MSE) between the different attenuation maps as compared to the gold standard (MTM) was also assessed. Well-established figures of merit known to have a large influence on many types of task performance are generally used to assess image quality. The metrics selected in our assessment, namely contrast and noise (uniformity), are used as measures of image quality. Absolute and relative quantification indexes were evaluated and the correlation between them checked. Statistical analysis was also performed to assess the significance of the differences. The relative difference between rCGM estimates using the different methods and MTM (gold standard) was defined as:

Percent difference $=\frac{V_{\text {method }}-V O I_{M T M}}{V O I_{M T M}} \times 100 \%$

Template-based quantification is a crucial step when performing comparative evaluation of quantitative estimates. T1-weighted 
standardised stereotactic MRI templates provided within the Statistical Parametric Mapping (SPM99) software package (Wellcome Department of Cognitive Neurology, University College London, London, UK) [24] were used to delineate manually a total of 20 bilateral volumes of interest (VOIs) covering the totality of cortical and subcortical structures [10]. PET and MRI templates supplied with SPM come already spatially normalised with respect to the Montreal Neurological Institute (MNI) reference space [25]. Therefore, it was found convenient to register the reconstructed images with respect to the PET template. To this end, the images were re-aligned to the standardised stereotactic template using a nine-parameter rigid body transformation for automated VOI quantification. Twenty VOIs were defined on the multislice MRI template and transformed as mentioned above to the coordinate space of the PET images to quantify FDG uptake.

Absolute quantitation. The 20 VOIs defined by an experienced neuropsychologist were superimposed on each co-registered study, resulting in a total of 200 VOIs for the ten patients studied. Oneway repeated ANOVA was used to test significant quantitation differences in rCGM for each VOI when applying the different attenuation correction methods under investigation. Within-group variance was accounted for in this way since the patients were chosen without any a priori criteria.

Relative quantitation. The derivation of physiological parameters from semi-quantitative measures through comparison with a disease-free or receptor-void region has enabled simplified but clinically achievable and highly relevant measurements. Cerebellar normalisation is commonly reported in studies dealing with Alzheimer's disease and receptor imaging [13, 14]. To assess the effect of the attenuation map on relative quantitation with respect to cerebellum as the reference region, the mean rCGM for each VOI was normalised to mean right and left cerebellar rCGM estimates. Similar correlation and statistical analysis was then performed. In addition, the contrast between the corpus callosum and lateral ventricle and uniformity within the cerebellum were also compared between the different methods. The idea of uniformity measurement within the cerebellum is to minimise the deviations due to statistical fluctuations and to measure only systematic variations due to different attenuation correction procedures. The contrast between the corpus callosum and lateral ventricle is of special importance in certain neurological pathologies and was therefore included in our assessment.

\section{Results}

\section{Qualitative assessment}

Representative planes (superior, middle and inferior) of attenuation maps generated using the different methods for a clinical brain PET study are shown in Fig. 1. It is worth noting the slight rotational misregistration between the transmission and emission (TX-EM) scans for this patient. In fact, the misregistration between emission and transmission scans is reflected by a clearly visible rotational misregistration between the MTM and ACDM attenuation maps shown in Fig. 1, the latter being computed from the emission scan. This misalignment is well recovered by both SMM and IADM procedures. The MRI-PET co-registration algorithm seems to produce smaller errors compared with TX-EM, even when a thermoplastic face mask is used for reproducible patient positioning. Non-uniformities of the attenuation map are obvious on planes containing sinus and air cavities. In theory, the advantages of transmission scanning are well established though segmented MRI appears to better resolve these structures. It was observed that the user-

Fig. 1. Three different transaxial planes of a patient study illustrating attenuation maps derived using the different methods. From top to bottom: superior, middle and inferior planes, and from left to right: UFEM, ACDM, MTM, STM, SMM and IADM

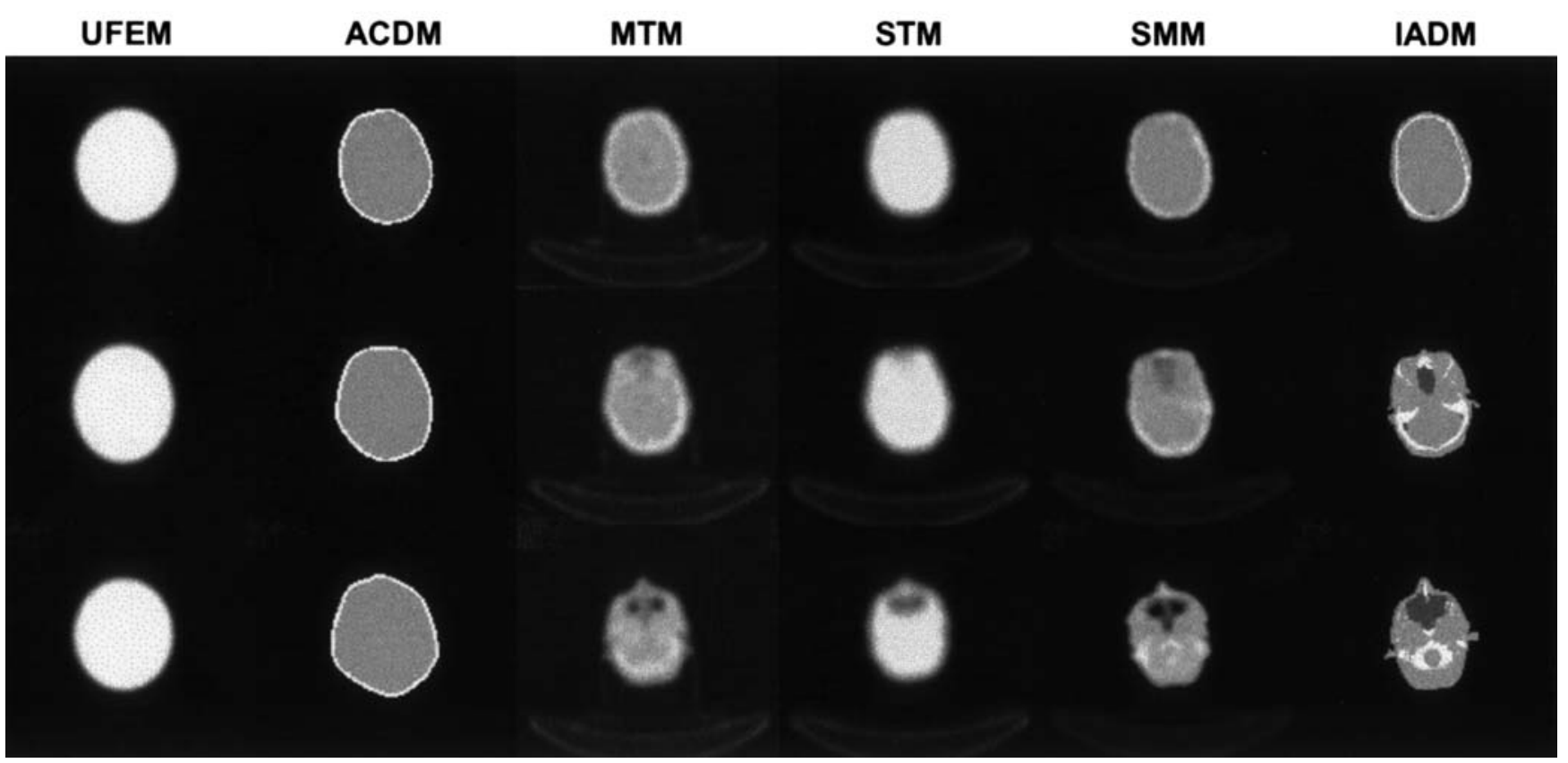




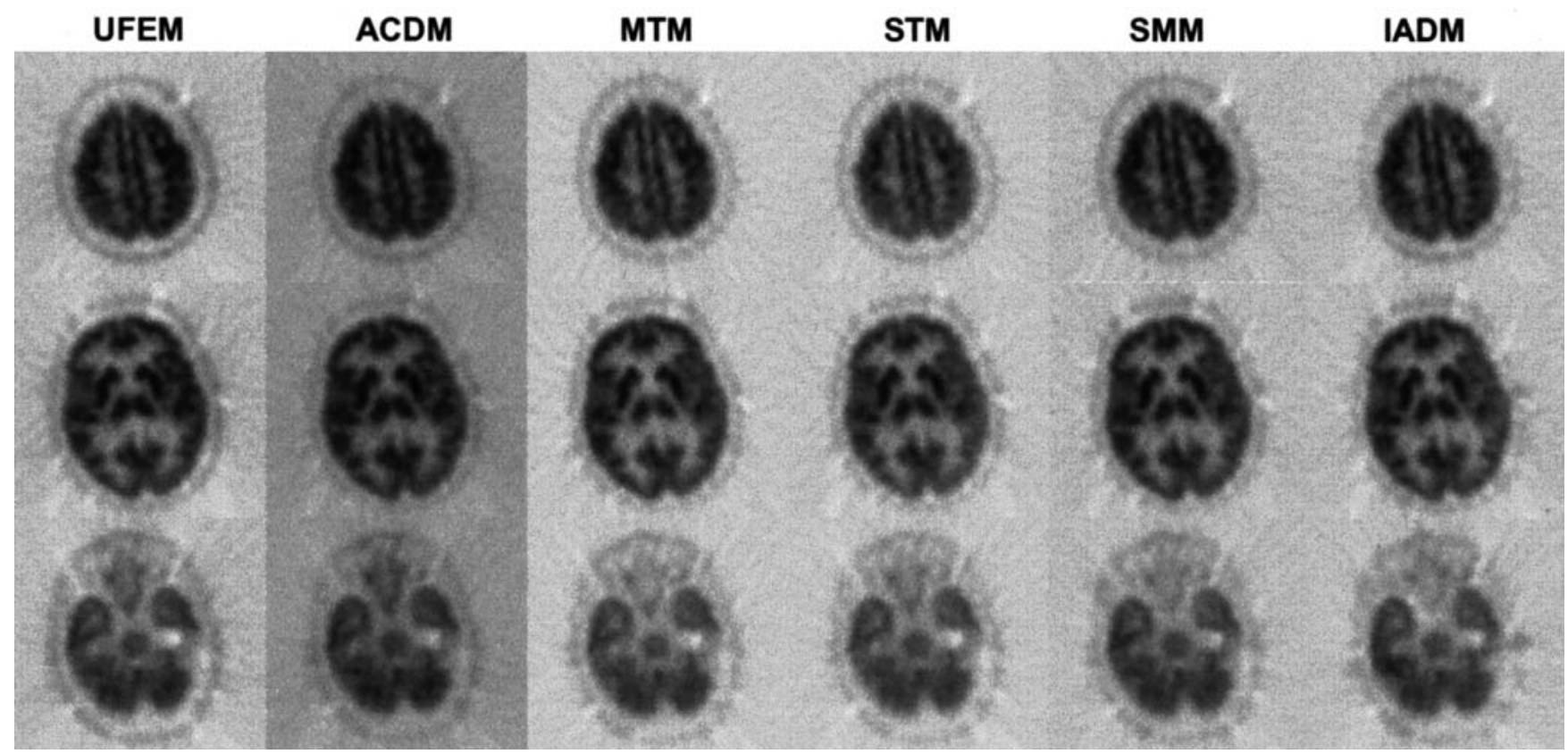

Fig. 2. Three different transaxial PET images of a patient study reconstructed using the different attenuation correction techniques. From top to bottom: superior, middle and inferior planes, and from left to right: UFEM, ACDM, MTM, STM, SMM and IADM

Fig. 3a-e. Horizontal profiles drawn across the middle transaxial image shown in Fig. 2 at the level of the thalamus, comparing reconstructions guided by MTM (solid line), and alternative attenuation correction methods (dashed line). a UFEM, b ACDM, c STM, d SMM and e IADM

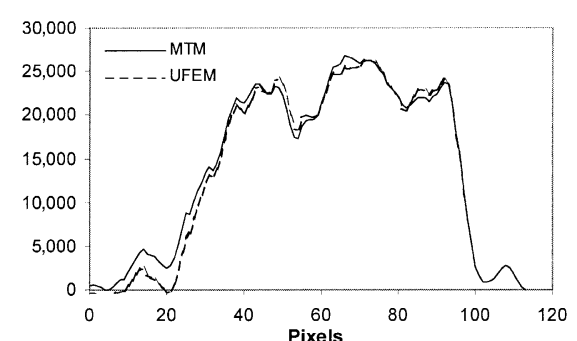

(a)

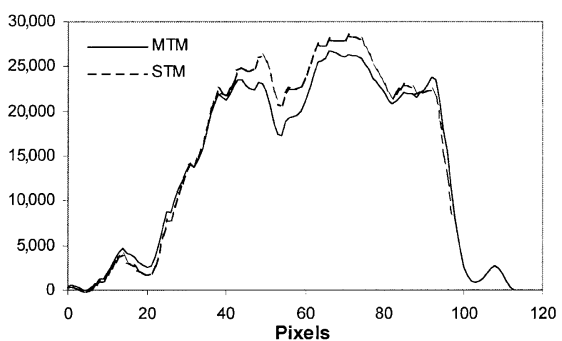

(c)

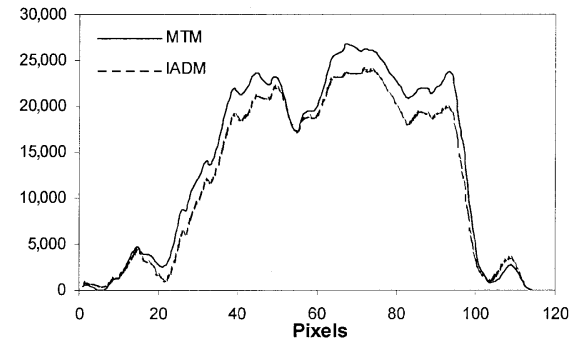

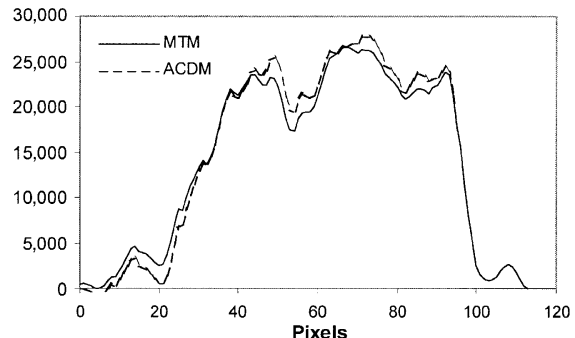

(b)

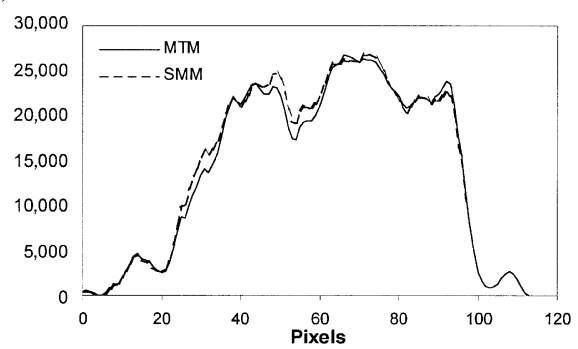

$(d)$ dependent UFEM method overestimates the head contour on the majority of slices. This is more pronounced in the case of ACDM, especially on the external planes. In addition, the assignment of theoretical attenuation coefficient of bone $\left(\mu=0.151 \mathrm{~cm}^{-1}\right)$ is not straightforward since smaller values are generally observed on clinical transmission scans $\left(\mu=0.136 \pm 0.08 \mathrm{~cm}^{-1}\right)$ [8].

Corresponding reconstructed PET images are illustrated in Fig. 2. The qualitative assessment was restricted to subjective observer rating scores performed by expert 


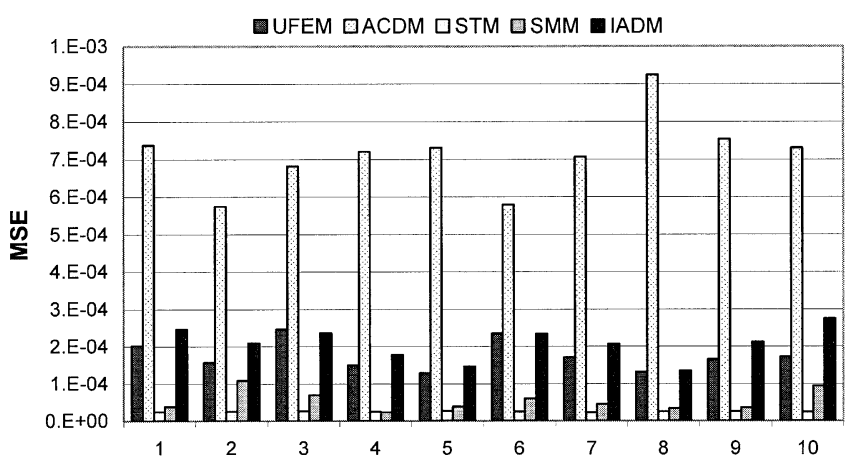

Fig. 4. Mean square errors (MSE) between the different attenuation maps as compared to the gold standard (MTM) for the ten patients studied

physicians. A merely qualitative analysis revealed the merits of the more exact methods based on realistic nonuniform attenuation maps. They produce less visible artefacts, while the approximate methods tend to produce an artefact in which there is a high level of activity along the edge of the image due to overestimation of the head contour when using ACDM on the outer slices. In addition, horizontal profiles drawn across the middle transaxial image shown in Fig. 2 at the level of the thalamus are shown in Fig. 3 to further illustrate the noise level and quantitative performance when comparing reconstructions guided by MTM and alternative attenuation correction schemes. The segmented transmission-based reconstruction method overestimates the activity concentration in the central part, with corresponding slight underestimation at the extremities (Fig. 3c). This might be explained by similar variation in values of attenuation coefficients between non-segmented and segmented attenuation maps [19]. More significant underestimation in the central part was observed in the case of IADM (Fig. 3e), which can be explained in part by the large sinus cavity in corresponding planes.

\section{Quantitative analysis}

Absolute quantitation. Figure 4 shows mean square errors (MSE) between the different attenuation maps as compared to MTM, considered as the "gold standard" for each of the ten patients studied in this work. The MSE between MTM and the different attenuation maps is larger when using ACDM owing to the fact that the current implementation of this method results in significant overestimation of the contours on the external slices. Correlation plots between average absolute rCGM values obtained from reconstructions of clinical 3D brain scans guided by MTM and alternative attenuation correction methods using a total of 200 data points are illustrated in Fig. 5. The line connecting the data points rep-
Fig. 5a-e. Correlation plots between mean absolute rCGM values obtained from reconstructions of clinical 3D brain scans guided by measured transmission and alternative attenuation correction methods. A total of 200 data points (ten patients and 20 VOIs) are shown. a UFEM and MTM, b ACDM and MTM, c STM and MTM, d SMM and MTM and e IADM and MTM. Correlation coefficients and best fit equations are also shown

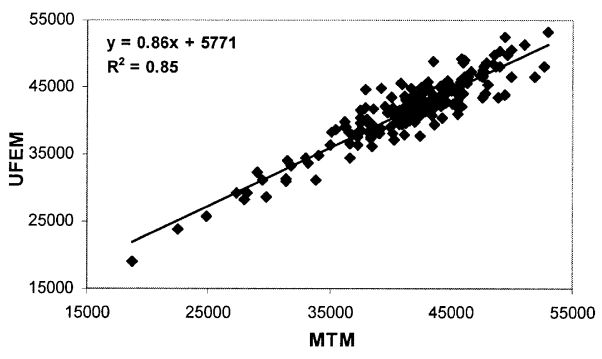

(a)

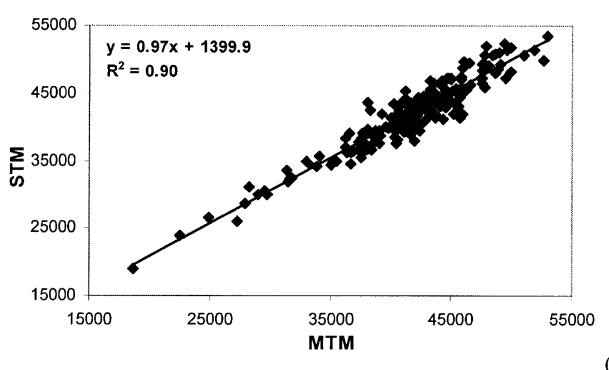

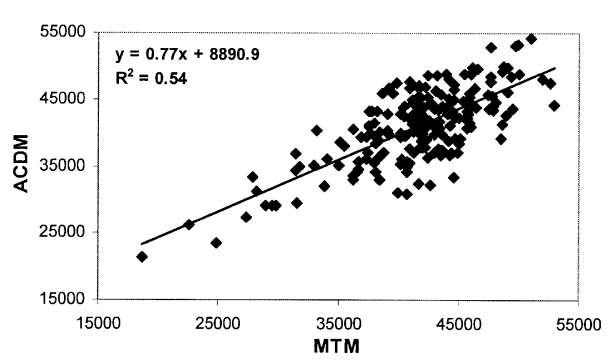

(b)

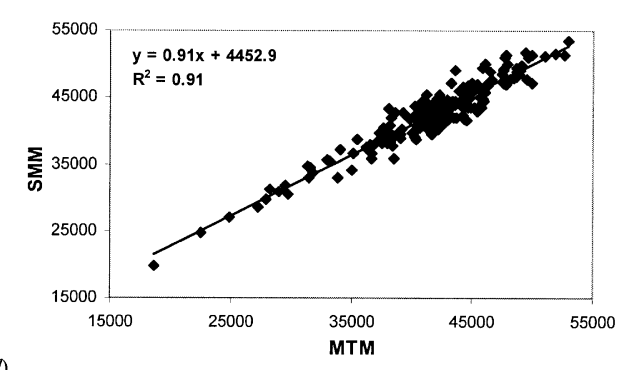

(d) (c)

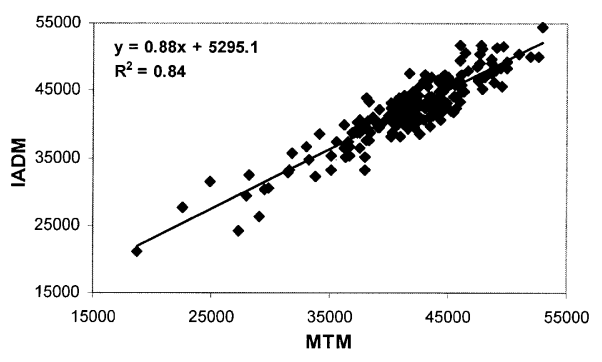

(e) 
Fig. 6a-e. Relative difference between absolute rCGM estimates for clinical 3D brain reconstructions when using the different attenuation correction techniques as compared to the gold standard (MTM). a UFEM, b ACDM, c STM, d SMM and e IADM. See Table 1 for abbreviations
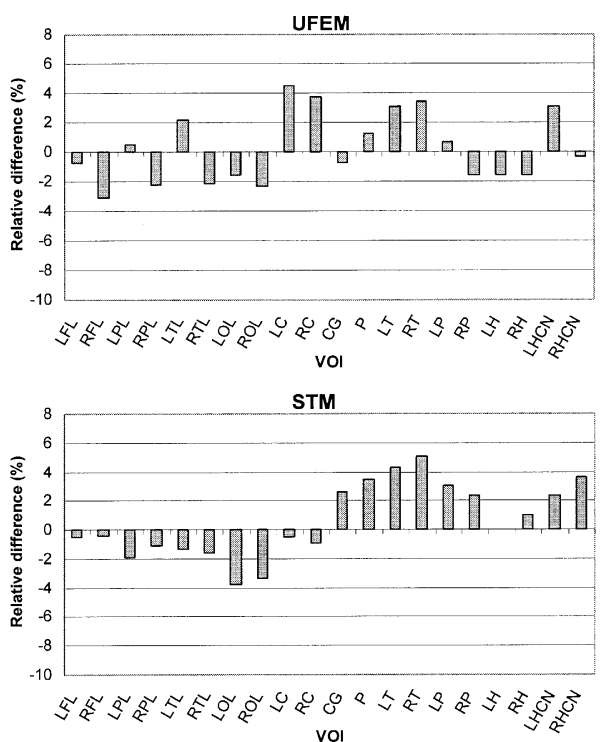

(c)

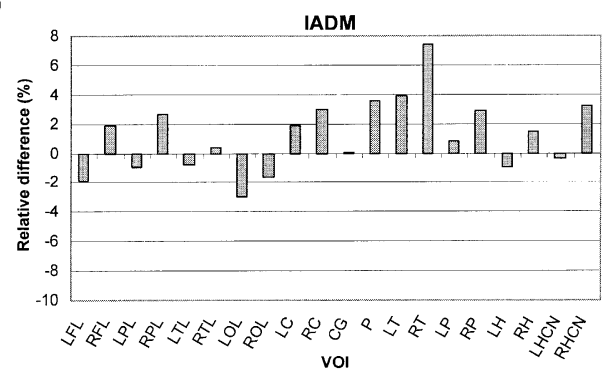

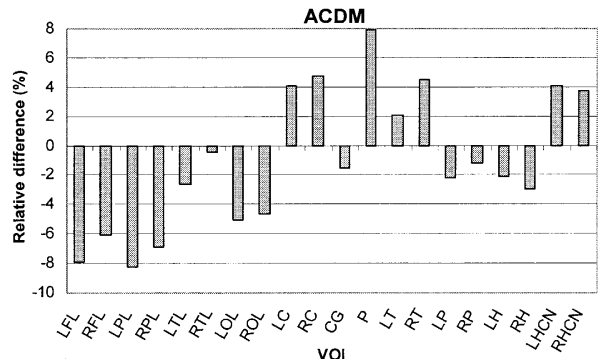

(b)

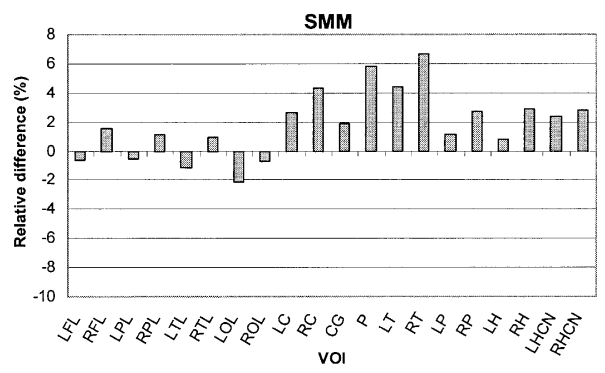

(d)

resents the results of linear regression analysis. Correlation between the mean regional cerebral glucose metabolism (rCGM) values obtained with the various attenuation correction methods and those obtained with the gold standard (MTM) was good, except in the case of ACDM $\left(R^{2}=0.54\right)$. The STM and SMM methods showed the best correlation $\left(R^{2}=0.90\right)$ and the regression lines agreed well with the line of identity. It is worth emphasising that the analysis of only 20 VOI data points belonging to a single patient followed the same pattern with respect to best- and worst-case regression. Figure 6 shows relative differences between absolute rCGM estimates for clinical 3D brain reconstructions when using different attenuation correction schemes as compared to MTM. Relative differences in mean rCGM values were in general less than $8 \%$. The difference in average rCGM values between UFEM and MTM varied between $0.3 \%$ and $4.5 \%$ for all VOIs.

The quantitative VOI-based analysis revealed different performance characteristics and statistically significant differences between the various correction techniques when compared to the gold standard (MTM) for some regions of the brain. Table 1 summarises the results obtained using repeated ANOVA analysis comparing absolute rCGM estimates obtained from clinical brain PET reconstructions. Metrics include ANOVA significance, paired sample correlation and paired sample significance (the last two are not shown). Overall, individual VOI standard deviations were significantly smaller for calculated and segmented-based attenuation correction algorithms compared with MTM. The pons and thalamus VOIs showed the highest probability of a true difference for both STM and SMM methods $(P \leq 0.0025)$, while right parietal and temporal lobe VOIs, as well as right thalamus and right head of caudate nucleus VOIs, had the highest correlation coefficients relating MTM and alternative attenuation correction schemes, except in the case of ACDM (Table 1).

Relative quantitation. Correlation for all VOIs and all patients between relative rCGM values normalised to the cerebellum obtained from reconstructions of clinical 3D brain scans guided by measured transmission and alternative attenuation correction schemes was found to be high (not shown). Box and whisker plots for assessment of contrast between the corpus callosum and the lateral ventricle and uniformity in the cerebellum when using different attenuation correction schemes are illustrated in Figs. 7 and 8, respectively. The analysis of contrast assessment between the corpus callosum and the lateral ventricle when using different attenuation correction schemes revealed significantly lower estimates when using MTM and STM, the former being noisier than all other methods. Although the reasons for the improve- 
Table 1. Summary of statistical assessment using repeated ANOVA analysis for comparison of absolute rCGM estimates obtained from clinical brain PET reconstructions based on different attenuation correction techniques as compared with reconstruc- tions guided by measured transmission (MTM), which served as the gold standard. Metrics shown were limited to ANOVA significance level corrected for multiple comparisons

\begin{tabular}{|c|c|c|c|c|c|}
\hline Volume of interest & UFEM & $\mathrm{ACDM}$ & STM & SMM & IADM \\
\hline Left frontal lobe (LFL) & 0.499 & 0.017 & 0.652 & 0.502 & 0.160 \\
\hline Right frontal lobe (RFL) & 0.078 & 0.057 & 0.659 & 0.191 & 0.123 \\
\hline Left parietal lobe (LPL) & 0.621 & 0.037 & 0.115 & 0.413 & 0.518 \\
\hline Right parietal lobe (RPL) & 0.108 & 0.039 & 0.318 & 0.323 & 0.213 \\
\hline Left temporal lobe (LTL) & 0.101 & 0.342 & 0.300 & 0.154 & 0.608 \\
\hline Right temporal lobe (RTL) & 0.139 & 0.868 & 0.076 & 0.488 & 0.794 \\
\hline Left occipital lobe (LOL) & 0.155 & 0.082 & $0.002^{\mathrm{a}}$ & 0.008 & 0.047 \\
\hline Right occipital lobe (ROL) & 0.068 & 0.145 & $0.002^{\mathrm{a}}$ & 0.517 & 0.323 \\
\hline Left cerebellum (LC) & 0.011 & 0.178 & 0.613 & 0.020 & 0.336 \\
\hline Right cerebellum (RC) & 0.036 & 0.137 & 0.271 & 0.007 & 0.118 \\
\hline Cingulate gyrus (CG) & 0.708 & 0.562 & 0.068 & 0.222 & 0.965 \\
\hline Pons $(\mathrm{P})$ & 0.469 & 0.032 & 0.003 & $0.001^{\mathrm{a}}$ & 0.135 \\
\hline Left thalamus (LT) & 0.110 & 0.499 & 0.003 & $0.002^{\mathrm{a}}$ & 0.023 \\
\hline Right thalamus (RT) & 0.047 & 0.137 & 0.005 & $<0.001^{\mathrm{a}}$ & $0.002^{\mathrm{a}}$ \\
\hline Left putamen (LP) & 0.622 & 0.464 & 0.051 & 0.190 & 0.605 \\
\hline Right putamen (RP) & 0.266 & 0.611 & 0.027 & 0.037 & 0.087 \\
\hline Left hippocampus (LH) & 0.316 & 0.422 & 0.982 & 0.454 & 0.478 \\
\hline Right hippocampus (RH) & 0.417 & 0.313 & 0.374 & 0.092 & 0.304 \\
\hline Left head of caudate nucleus (LHCN) & 0.157 & 0.177 & 0.114 & 0.053 & 0.864 \\
\hline Right head of caudate nucleus (RHCN) & 0.871 & 0.197 & 0.004 & 0.009 & 0.028 \\
\hline
\end{tabular}

a Regions showing highest probability of a true difference

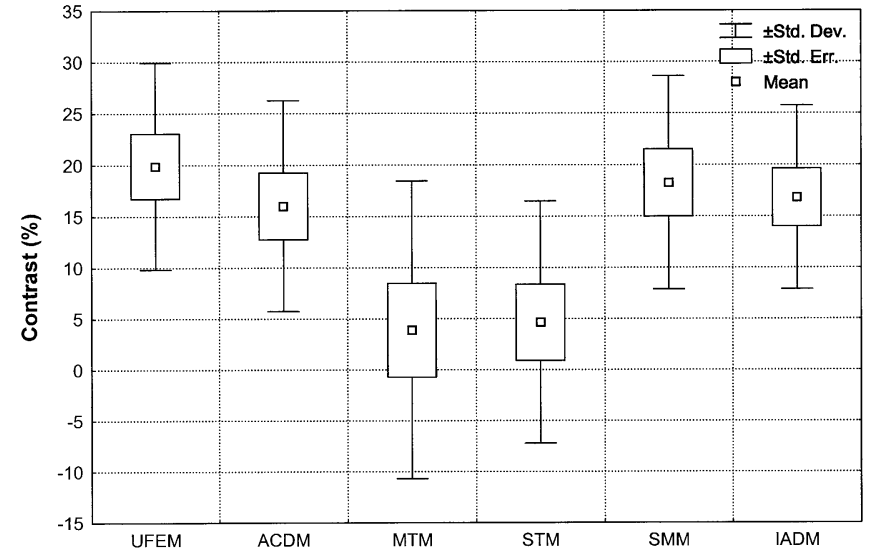

Fig. 7. Box and whisker plot for contrast assessment between the corpus callosum and the lateral ventricle when using different attenuation correction schemes

ment in the contrast when using non-uniform attenuation maps derived from either Atlas or patient-specific MR images seem obvious, the explanation for this improvement when using approximate methods (UFEM and ACDM) is not clear. No significant differences were noticed with respect to uniformity assessment when using the different attenuation correction schemes investigated in this study. Again, MTM exhibited the highest SDs, which can be explained by significant propagation of sta-

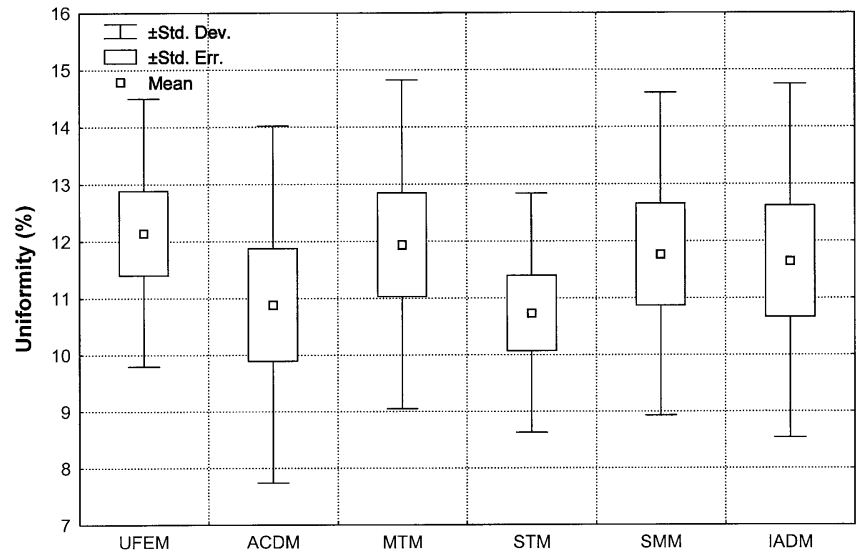

Fig. 8. Box and whisker plot for uniformity assessment in the cerebellum when using the different attenuation correction techniques

tistical noise from short transmission scans to emission data.

The results of statistical analysis comparing relative rCGM estimates obtained from clinical brain PET reconstructions based on different attenuation correction schemes as compared to reconstructions guided by measured transmission are summarised in Table 2. The number of VOIs showing a high probability of a true difference increased substantially after normalisation to the 
Table 2. Summary of statistical assessment using repeated ANOVA analysis for comparison of relative rCGM estimates obtained from clinical brain PET reconstructions based on different attenuation correction techniques as compared with reconstructions guid-

\begin{tabular}{|c|c|c|c|c|c|}
\hline Volume of interest & UFEM & ACDM & STM & SMM & IADM \\
\hline Left frontal lobe (LFL) & $<0.001^{\mathrm{a}}$ & $<0.001^{\mathrm{a}}$ & 0.952 & $<0.001^{\mathrm{a}}$ & 0.017 \\
\hline Right frontal lobe (RFL) & $<0.001^{\mathrm{a}}$ & $<0.001^{\mathrm{a}}$ & 0.846 & 0.029 & 0.668 \\
\hline Left parietal lobe (LPL) & 0.006 & $0.002^{\mathrm{a}}$ & 0.140 & $0.002^{\mathrm{a}}$ & 0.074 \\
\hline Right parietal lobe (RPL) & $<0.001^{\mathrm{a}}$ & $<0.001^{\mathrm{a}}$ & 0.661 & 0.006 & 0.942 \\
\hline Left temporal lobe (LTL) & 0.110 & $<0.001^{\mathrm{a}}$ & 0.440 & $<0.001^{\mathrm{a}}$ & 0.021 \\
\hline Right temporal lobe (RTL) & $<0.001^{\mathrm{a}}$ & $0.002^{\mathrm{a}}$ & 0.250 & $0.001^{\mathrm{a}}$ & 0.201 \\
\hline Left occipital lobe (LOL) & $<0.001^{\mathrm{a}}$ & $<0.001^{\mathrm{a}}$ & $<0.001^{\mathrm{a}}$ & $<0.001^{\mathrm{a}}$ & 0.003 \\
\hline Right occipital lobe (ROL) & $<0.001^{\mathrm{a}}$ & $<0.001^{\mathrm{a}}$ & $0.002^{\mathrm{a}}$ & $<0.001^{\mathrm{a}}$ & 0.022 \\
\hline Left cerebellum (LC) & 0.470 & 0.533 & 0.260 & 0.069 & 0.200 \\
\hline Right cerebellum (RC) & 0.470 & 0.583 & 0.289 & 0.058 & 0.203 \\
\hline Cingulate gyrus (CG) & 0.013 & $0.002^{\mathrm{a}}$ & 0.017 & 0.187 & 0.149 \\
\hline Pons $(\mathrm{P})$ & 0.070 & 0.033 & $<0.001^{\mathrm{a}}$ & 0.027 & 0.604 \\
\hline Left thalamus (LT) & 0.393 & 0.094 & $<0.001^{\mathrm{a}}$ & 0.339 & 0.346 \\
\hline Right thalamus (RT) & 0.570 & 0.877 & $<0.001^{\mathrm{a}}$ & $<0.001^{\mathrm{a}}$ & 0.006 \\
\hline Left putamen (LP) & 0.040 & $0.002^{\mathrm{a}}$ & 0.003 & $0.002^{\mathrm{a}}$ & 0.187 \\
\hline Right putamen (RP) & $<0.001^{\mathrm{a}}$ & $<0.001^{\mathrm{a}}$ & $<0.001^{\mathrm{a}}$ & 0.132 & 0.805 \\
\hline Left hippocampus (LH) & $0.001^{\mathrm{a}}$ & $<0.001^{\mathrm{a}}$ & 0.386 & $0.001^{\mathrm{a}}$ & 0.063 \\
\hline Right hippocampus (RH) & 0.004 & 0.001 & 0.085 & 0.597 & 0.593 \\
\hline Left head of caudate nucleus (LHCN) & 0.582 & 0.685 & 0.017 & 0.078 & 0.125 \\
\hline Right head of caudate nucleus (RHCN) & 0.060 & 0.590 & $<0.001^{\mathrm{a}}$ & 0.331 & 0.753 \\
\hline
\end{tabular}

a Regions showing highest probability of a true difference

cerebellum for all methods except IADM. Nevertheless, higher correlation coefficients were obtained on semiquantitative estimates.

\section{Discussion}

A clinical diagnosis from PET is usually determined by visual inspection or semi-automated methods combined with absolute or semi-quantitative estimates of parameters of clinical interest. The impact of the attenuation map on image quality and quantitative analysis of SPET data, as well as differences between the effectiveness of uniform versus non-uniform attenuation maps, has been extensively studied $[2,11,12,13,14]$. Surprisingly, however, very few studies have addressed this issue using 3D PET images. More importantly, published results have generally been limited to conventional calculated versus transmission-based attenuation correction techniques implemented within software provided by scanner manufacturers $[3,4,26]$. Continuous efforts to develop robust approaches for determination of the attenuation map in brain PET imaging without transmission scanning have resulted in the proposal of several methods, including the inferring-attenuation distributions method based on deformable registration with respect to the brain component of a digital head atlas [9], the automated three-component method [8], and determination of the patient-specific head contour using an optical tracking ed by measured transmission (MTM), which served as the gold standard. Metrics shown were limited to ANOVA significance level corrected for multiple comparisons 
further motivates the improvement of this approach. In addition to the extra radiation absorbed dose to staff and patients when transmission scanning is performed, the choice of this method for determination of the patientspecific attenuation map has direct consequences for the total acquisition time, PET camera time planning and hence patient throughput.

There was a very good correlation $\left(R^{2}=0.90\right)$ between rCGM estimates with all attenuation correction techniques and that with MTM, and the regression lines agreed well with the line of identity except in the case of $\operatorname{ACDM}\left(R^{2}=0.54\right)$. Similar observations were made regarding ACDM with respect to the dispersion of data points, which was insignificant for all other techniques. In addition, the general trend as shown by the regression lines was that the coefficients of variation were similar with the exception of ACDM. However, it should be noted that ACDM led to $1 \%$ lower overall estimates than MTM, whereas SMM led to $1.9 \%$ higher estimates. A slightly higher correlation coefficient was obtained when using SMM while the regression line agreed better with the line of identity when using STM on both absolute and relative quantitative estimates (Fig. 5). The poorest correlation was observed for ACDM in both cases. The choice of the optimal attenuation correction procedure could have important clinical consequences. Van Laere et al. [2] reported that transmission-based attenuation correction produces a slight increase in inter-subject variability. Since our study encompassed a limited set of clinical data, further research is essential in various patient populations to establish the full clinical impact of the different attenuation correction techniques assessed in this work.

Different approaches have been suggested to judge image quality when evaluating image correction and reconstruction algorithms. In the simplest approach, trained nuclear medicine physicians carry out observer performance studies and are asked to rank images by their degree of quality. A common method for assessment of image quality with respect to a detection task is the use of observer studies, where the performance of any observer (human or computer algorithm) is characterised by receiver operating characteristics (ROC) analysis. In such studies, observers rate images based on their confidence that a defect/lesion exists in a large set of images. Curve-fitting methods are used to fit the rating data to ROC curves, which plot the co-variation in "true positive" and "false positive" conditional probabilities summarising how accurately those image ratings could be used to separate the actually abnormal cases from the normal cases [29]. The estimated area under the fitted ROC curve is often used as a general index of image quality or performance accuracy for any alternative classification task. ROC and localisation ROC (LROC) techniques have been extensively used to evaluate lesion detectability. However, such techniques rely on experimental phantom measurements or simulated data since the ground truth needs to be known. Therefore, our qualitative assessment was restricted to subjective observer rating scores by two experienced physicians. An interesting approach in future comparative evaluation studies would be to carry out voxel-based statistical analysis using SPM. Our recent study on the impact of model-based scatter correction and iterative reconstruction on spatial distribution of ${ }^{18} \mathrm{~F}-\mathrm{FDG}$ in reconstructed brain PET images of healthy subjects using this kind of analysis demonstrated that iterative reconstruction does not result in significant changes when compared with analytic reconstruction procedures, while significant differences in ${ }^{18} \mathrm{~F}$ FDG distribution arise when images are reconstructed with and without explicit scatter correction for some cerebral areas [30]. This needs to be acknowledged for adequate interpretation of 3D brain PET images after applying scatter correction.

One of the motivations for the development of SMM is to achieve accurate quantitative accuracy with a transmission-less brain PET tomograph. Removal of the transmission scanning component simplifies greatly the complexity of the tomograph's design and acquisition protocols and contributes significantly to lowering the cost of the prototype. In fact, our group is involved within the Computed Imaging for Medical Applications (CIMA) collaboration in the design of a novel and innovative high-resolution Compton-enhanced 3D brain PET tomograph dedicated to brain research (patent applied for, PCT/EP 02/07967) [31]. This concept leads to an image reconstruction which is free of any parallax error and provides a uniform spatial and energy resolution over the whole sensitive volume. The key components are a matrix of long scintillator crystals and Hybrid Photodiodes (HPD) with matched segmentation and integrated readout electronics.

It is recognised that this study included a restricted set of patients and did not encompass experimental phantom studies for detailed characterisation of the different methods in a controlled manner. The use of phantom studies where ground truth can be established would allow for a quantitative understanding of the source and magnitude of errors in the attenuation and emission data. Future work will concentrate on similar studies using a fully tissue-equivalent anthropomorphic head phantom manufactured by RSD (Radiology Support Devices, Long Beach, CA) when it becomes available in our department. Nevertheless, the segmented MRI-guided attenuation correction technique cannot be evaluated using such a phantom. Another aspect that deserves further attention with emerging applications of dual-modality PET/CT imaging devices in clinical routine is the investigation of the limitations of CT-based attenuation correction in the presence of metallic dental implants, which are known to introduce significant artefacts not only on CT but also on radionuclide transmission scans and therefore the reconstructed brain PET images [32]. Investigation of the potential of the different methods in- 
vestigated in this study as compared to the CT-based approach is strongly needed.

\section{Conclusion}

The attenuation map influences both absolute and relative quantitation in cerebral 3D PET. The quantitative VOI-based analysis revealed different performance characteristics and statistically significant differences between the different attenuation correction techniques when compared with the gold standard (MTM). Transmission-less attenuation correction results in a reduced radiation dose and makes a dramatic difference in acquisition time. The necessity of acquiring an additional TX scan is a restrictive factor for patient throughput. Considering the difficulties associated with TX-based attenuation correction and the limitations of the current calculated attenuation correction methods, MRI-guided attenuation correction in 3D brain PET would likely be the method of choice for the foreseeable future as the best approach in a brain PET research facility and could be applied to other functional brain imaging modalities (e.g. SPET). This might also have consequences for future designs of PET cameras dedicated to brain research, where addition of a transmission scanning component will possibly be unnecessary, thereby simplifying the complexity of the tomograph's design and lowering its cost [31].

Acknowledgements. This work was supported by the Swiss National Science Foundation under grants SNSF 3152-062008 and 3152A0-102143. The authors would like to thank Manuel DiazGomez for performing the analysis of the data sets.

\section{References}

1. Zaidi H, Hasegawa BH. Determination of the attenuation map in emission tomography. J Nucl Med 2003; 44:291-315.

2. Van Laere K, Koole M, Versijpt J, Dierckx R. Non-uniform versus uniform attenuation correction in brain perfusion SPET of healthy volunteers. Eur J Nucl Med 2001; 28:90-98.

3. Setani K, Schreckenberger M, Sabri O, Meyer PT, Zeggel T, Bull U. Comparison of different methods for attenuation correction in brain PET: effect on the calculation of the metabolic rate of glucose [in German]. Nuklearmedizin 2000; 39:50-55.

4. Zaidi H, Laemmli C, Allaoua M, Gries P, Slosman DO. Optimizing attenuation correction in clinical cerebral 3D PET: which method to use? J Nucl Med 2001; 42:195-196.

5. Zaidi H, Montandon M-L. Which attenuation coefficient to use in combined attenuation and scatter corrections for quantitative brain SPET? Eur J Nucl Med Mol Imaging 2002; 29:967-969.

6. Zaidi H, Sossi V. Correction for image degrading factors is essential for accurate quantification of brain function using PET. Med Phys 2003; in press.

7. Bergstrom M, Litton J, Eriksson L, Bohm C, Blomqvist G. Determination of object contour from projections for attenuation correction in cranial positron emission tomography. J Comput Assist Tomogr 1982; 6:365-372.
8. Weinzapfel BT, Hutchins GD. Automated PET attenuation correction model for functional brain imaging. J Nucl Med 2001; 42:483-491.

9. Stodilka RZ, Kemp BJ, Prato FS, Kertesz A, Kuhl D, Nicholson RL. Scatter and attenuation correction for brain SPECT using attenuation distributions inferred from a head atlas. J Nucl Med 2000; 41:1569-1578.

10. Zaidi H, Montandon M-L, Slosman DO. Magnetic resonance imaging-guided attenuation correction in 3D brain positron emission tomography. Med Phys 2003; 30:937-948.

11. Iida H, Narita Y, Kado H, et al. Effects of scatter and attenuation correction on quantitative assessment of regional cerebral blood flow with SPECT. J Nucl Med 1998; 39:181-189.

12. Arlig A, Gustafsson A, Jacobsson L, Ljungberg M, Wikkelso C. Attenuation correction in quantitative SPECT of cerebral blood flow: a Monte Carlo study. Phys Med Biol 2000; 45: 3847-3859.

13. Stodilka RZ, Kemp BJ, Prato FS, Nicholson RL. Importance of bone attenuation in brain SPECT quantification. $\mathrm{J} \mathrm{Nucl}$ Med 1998; 39:190-197.

14. Licho R, Glick SJ, Xia W, Pan TS, Penney BC, King MA. Attenuation compensation in ${ }^{99 m}$ Tc SPECT brain imaging: a comparison of the use of attenuation maps derived from transmission versus emission data in normal scans. J Nucl Med 1999; 40:456-463.

15. Nicholson R, Doherty M, Wilkins K, Prato F. Paradoxical effects of the skull on attenuation correction requirements for brain SPECT. J Nucl Med 1988; 29:1316.

16. Turkington TG, Gilland DR, Jaszczak RJ, Greer KL, Coleman RE, Smith MF. A direct measurement of skull attenuation for quantitative SPECT. IEEE Trans Nucl Sci 1993; 40:1158-1161.

17. Zaidi H, Montandon M-L, Slosman D. Impact of the attenuation map on relative and absolute quantitation in $3 \mathrm{D}$ brain PET: assessment of 6 different methods. Conference proceedings of the VIIth International Meeting on Fully Three-dimensional Image Reconstruction in Radiology and Nuclear Medicine, 29 June-4 July 2003, Saint-Malo, France. Available on CDROM.

18. Watson CC, Jones W, Brun T, Baker K, Vaigneur K, Young J. Design and performance of a single photon transmission measurement for the ECAT ART. Proc IEEE Nuclear Science Symposium and Medical Imaging Conference 1997; 2:13661370.

19. Zaidi H, Diaz-Gomez M, Boudraa AE, Slosman DO. Fuzzy clustering-based segmented attenuation correction in wholebody PET imaging. Phys Med Biol 2002; 47:1143-1160.

20. Xu M, Cutler P, Luk W. An adaptive local threshold segmented attenuation correction method for whole-body PET imaging. IEEE Trans Nucl Sci 1996; 43:331-336.

21. Woods RP, Grafton ST, Watson JD, Sicotte NL, Mazziotta JC. Automated image registration. II. Intersubject validation of linear and nonlinear models. J Comput Assist Tomogr 1998; 22:153-165.

22. Zubal IG, Harrell CR, Smith EO, Rattner Z, Gindi G, Hoffer BP. Computerized 3-dimensional segmented human anatomy. Med Phys 1994; 21:299-302.

23. Watson CC. New, faster, image-based scatter correction for 3D PET. IEEE Trans Nucl Sci 2000; 47:1587-1594.

24. Friston K, Holmes A, Worsley K, Poline J, Frith C, Frackwiak R. Statistical parametric maps in functional imaging: a general linear approach. Hum Brain Mapp 1995; 2:189-210.

25. Evans AC, Collins DL, Mills SR, Brown ED, Kelly RL, Peters TM. 3D statistical neuroanatomical models from 305 MRI 
volumes. IEEE Conference Record of Nuclear Science Symposium and Medical Imaging Conference 1993; 3:1813-1817.

26. Hooper PK, Meikle SR, Eberl S, Fulham MJ. Validation of postinjection transmission measurements for attenuation correction in neurological FDG-PET studies. J Nucl Med 1996; 37:128-136.

27. Watabe H, Sato N, Deloar HM, Urayama SI, Oka H, Iida H. Acquisition of attenuation map for brain PET study using optical tracking system. Proc. IEEE Nuclear Science Symposium and Medical Imaging Conference, San Diego, CA, 4-10 October, 2001. 3:1458-1461.

28. Kinahan PE, Townsend DW, Beyer T, Sashin D. Attenuation correction for a combined 3D PET/CT scanner. Med Phys 1998; 25:2046-2053.

29. Swensson RG. Unified measurement of observer performance in detecting and localizing target objects on images. Med Phys 1996; 23:1709-1725.
30. Montandon M-L, Slosman DO, Zaidi H. Assessment of the impact of model-based scatter correction on ${ }^{18} \mathrm{~F}-[\mathrm{FDG}] 3 \mathrm{D}$ brain PET in healthy subjects using statistical parametric mapping. Neuroimage 2003; in press.

31. Braem A, Chesi E, Joram C, Garibaldi F, Joram C, Mathot S, Nappi E, Schoenahl F, Séguinot J, Weilhammer P, Zaidi H. Novel design of high-resolution parallax-free Compton enhanced PET scanner dedicated to brain research. Conference proceedings of the First International Meeting on Applied Physics, 15-18th October 2003. Badajoz, Spain; 2003:in press.

32. Goerres GW, Hany TF, Kamel E, von Schulthess GK, Buck A. Head and neck imaging with PET and PET/CT: artefacts from dental metallic implants. Eur J Nucl Med Mol Imaging 2002; 29:367-370 